Indem die Rolle und Fähigkeit, Weltversionen zu erzeugen, primär an den Menschen gebunden wird, kann der Mensch bereits als Medium der Welterzeugung konzipiert werden. Er ist es, der seit Anbeginn seiner Geschichte aktiv am Gestaltungs- und Kommunikationsprozess mitwirkt und die Kultur- und Menschheitsgeschichte prägt. ${ }^{6}$ Der Medienwissenschaftler Werner Faulstich (1998; id. 2002) grenzt dieses sogenannte Menschmedium (z. B. Erzähler*in, aber auch Feste, Tänze oder Hinrichtungsrituale) von Gestaltungs- (z. B. Statuen, Skulpturen, Denkmäler, Architektur) und Schreibmedien (z. B. Brief, Flugblatt, Zeitschrift) ab. Allerdings darf nicht vergessen werden, dass es auch der Mensch ist, der Gestaltungs- und Schreibmedien hervorbringt, und somit in der Rolle des Produzenten und Trägers nicht aufhört, Medium der Vermittlung von Erfahrungen ${ }^{7}$ und Ereignissen ${ }^{8}$ zu sein.

\title{
2.2 NarRative Wissens- Und Welterzeugung ${ }^{9}$
}

Insbesondere Mensch- und Schreibmedien verwenden die menschliche Sprache als arbiträres und konventionalisiertes Symbol- und Zeichensystem (Saussure), um zeitlich

6 Die grundlegende Bedeutung von zwischenmenschlicher Kommunikation für die Entstehung von individuellem wie kollektivem Bewusstsein und Gedächtnis - verstanden als Wissensbestände über sich selbst und die Gruppen, denen ein Mensch angehört - wurde in der Vergangenheit ausführlich hervorgehoben und in Konzepten wie denen der sozialen Rahmungen und des kollektiven Gedächtnisses (Halbwachs), des kulturellen und kommunikativen Gedächtnisses (Assmann, Welzer) oder der Erinnerungsorte (Nora) festgehalten.

7 Der Sozialwissenschaftler Alfred Schütz konzipiert den Unterschied zwischen ,erleben “ und ,erfahren' sowie die Transformation vom Erleben zum Erfahren folgendermaßen: Während des Vorgangs des Erlebens verarbeitet der Körper ständig sinnliche Eindrücke, die allerdings nicht bewusst wahrgenommen werden und nicht reflexiv zugänglich sind. Erleben ist somit ein vorreflexiver Vorgang, wie das Zuhören und Mitschreiben eines Vortrags. Wenn diese Erlebnisse zu einem Gedankeninhalt, also zu etwas bewusst Wahrgenommenem werden, über das nachgedacht und gesprochen werden kann, transformieren sie sich zu Erfahrungen (cf. Abels 2001, 62).

8 Aus narratologischer Sicht sind Ereignisse weder etwas Gegebenes noch etwas Natürliches, sondern vielmehr das Ergebnis bestimmter Arten der Welterzeugung, die mit Selektion, Löschung, Abstraktion und Priorisierung zu tun haben. Die Narratologie bietet eine Reihe nützlicher Kriterien, um den Begriff ,Ereignis‘ zu definieren, indem man ihn vom Begriff, Geschehen' abgrenzt und verschiedene Grade von ,Ereignishaftigkeit' unterscheidet. Anknüpfend an die alltägliche Bedeutung von Ereignis als ,signifikanter Vorfall` oder ,signifikantes Ereignis‘ unterscheidet die Erzähltheorie zunächst zwischen der Gesamtheit aller Ereignisse und dem Ereignis als besonders relevantem und wesentlichem Teil davon. Die Beschaffenheit eines Ereignisses rührt daher, dass es aus dem kontinuierlichen Fluss von Ereignissen herausgehoben wird und dadurch als etwas Besonderes oder Überraschendes qualifiziert wird: Es basiert also auf der Auswahl und Unterscheidung durch den/die Beobachter*in (cf. Hühn 2012; Nünning 2010, 197-199).

$9 \mathrm{Zu}$ den unterschiedlichen Positionen und Einschätzungen des Verhältnisses von Literatur, Kunst, Fotografie und Film zu Realität/Wirklichkeit vom 18. bis zum 21. Jahrhundert siehe 
zurückliegende Erfahrungen und Ereignisse (mündlich oder schriftlich) zu codieren und zu vermitteln. ${ }^{10} \mathrm{Um}$ aus den gesammelten Erfahrungen und Ereignissen ein kohärentes Ganzes zu machen, das über einen längeren Zeitraum - für ein Individuum oder eine Gruppe - erinnerbar bleibt, entwickelte der Mensch die Fähigkeit des Erzählens, das in der Erzähltheorie mittlerweile als ,,anthropologisches Grundbedürfnis des Menschen“ (Nünning A. 2013, 18) beziehungsweise als ,anthropologische Universalie“ (Scheffel 2004, 121; Köppe/Kindt 2014, 13) angesehen wird. Aus evolutionsgeschichtlicher Sicht ist Erzählen als eine „Methode des ,Verschnürens“ von Informationen“"11 (Scheffel 2011, 76) zu verstehen, mit der diese in einen kausalen Bezug zueinander gebracht werden - oder, im Sinne von Goodman, in Beschreibungsprozessen komponiert, gewichtet, geordnet, getilgt oder ergänzt und deformiert werden -, um die Informationen zu speichern. Um aus literaturwissenschaftlicher Sicht von einer Erzählung im weitesten Sinne sprechen zu können, müssen mindestens zwei Informationen, die in der Literaturwissenschaft meist als ,Ereignisse“ bezeichnet werden, temporal geordnet und sinnhaft miteinander verknüpft sein. Mit anderen Worten, die Ereignisse müssen zeitlich verortet sein und einen kausalen Bezug zueinander aufweisen, womit die Ereignisse die Handlung einer Erzählung ausmachen (cf. Köppe/Kindt 2014, 50-64). ${ }^{12}$

die systematisch und analytisch aufbereitete Datenbank mit zentralen Texten von Jean Baudrillard über Immanuel Kant und Marshall McLuhan bis zu Virginia Woolf unter der Leitung von Susanne Knaller (2013) Realität und Wirklichkeit in der Moderne.

10 Produktion und Zirkulation des gesellschaftlich geteilten Wissensschatzes erfolgen primär über das Zeichensystem der Sprache (cf. Berger/Luckmann 1991, 51): „Language objectivates the shared experiences and makes them available to all within the linguistic community, thus becoming both the basis and the instrument of the collective stock of knowledge. Furthermore, language provides the means for objectifying new experiences, allowing their incorporation into the already existing stock of knowledge, and it is the most important means by which the objectivated and objectified sedimentations are transmitted in the tradition of the collectivity in question“ (ibid., 85-86).

11 Diese Fähigkeit ist ,auf die Entwicklung eines Zeichensystems angewiesen“ (Scheffel 2011, 76), deren Prototyp das verbale Erzählen ist.

12 Strukturbestimmende Charakteristika des Erzählens hat bereits Aristoteles in seiner Poetik aufgestellt, wo er zu den charakteristischen Merkmalen einer Erzählung die Mimesis [1], die Katharsis [2] und die Dramaturgie [3] zählt: „[1] Mimesis: In einer Erzählung werden menschliche Handlungen und Erfahrungen dargestellt bzw. nachgeahmt. Diese Darstellung bzw. Nachahmung menschlicher Handlungen und Erfahrungen nennt Aristoteles Mimesis (= erzählerische Nachahmung des Geschehens). Die Darstellung bzw. Nachahmung kann sich dabei auf die Vergangenheit (= Rekonstruktion, Bewahrung), die Gegenwart (=Verstehen, Reflexion) oder die Zukunft (=Entwurf, Imagination) beziehen. [2] Katharsis: Zweck der Mimesis ist die Katharsis, d. h. die emotionale Läuterung des Zuhörers. Erzählungen gehen somit in der Regel mit Gefühlen wie Spannung, Mitgefühl, Freude oder Trauer einher. [3] Dramaturgie: Eine Erzählung besitzt einen Anfang, eine Mitte und ein Ende. Die bloße Aneinanderreihung von Handlungen und Erfahrungen stellt somit noch keine Erzählung dar. Erzählungen benötigen vielmehr eine Dramaturgie, d. h. eine dramatische Form, durch die sie in ihren einzelnen Elementen zusammengehalten werden. Am Anfang werden dazu die handelnden Personen (= dramatis personae) sowie das Thema, bzw. das Motiv eingeführt. 
$\mathrm{Zu}$ dieser Minimaldefinition können weitere Strukturbestimmungen wie Ereignisreferenz, Handlungsabgeschlossenheit und ,tellability“ (Erzählwürdigkeit) hinzugefügt werden, die zu komplexeren Erzählungen führen. Die Bedingung der Ereignisreferenz „besteht darin, dass in Erzählungen Ereignisse auf eine Weise beschrieben werden, die diese zu den Belangen von Personen, also etwa ihren Bedürfnissen, Hoffnungen oder Meinungen, in Beziehung setzt“ (ibid., 64). Die Handlungsabgeschlossenheit einer Erzählung signalisiert ihren dramaturgischen Aufbau in Anfang - Mitte - Ende, der bereits bei Aristoteles Erwähnung findet. Mit ,tellability“ ist das gemeint, was eine Person an bestimmten Ereignissen für mitteilungswürdig und erzählenswert erachtet. Durch das Erzählen wird somit ein Informationsdefizit beseitigt und das Publikum emotional involviert. Für die weitere wie engere Begriffsbestimmung der Erzählung gilt, dass eine Erzählung „die für uns erfahrbare Wirklichkeit strukturiert“ (ibid., 71), denn sehr oft beziehen sich die Inhalte von Erzählungen auf konkrete Ereignisse aus unserer Lebenswelt.

Die Kultur- und Literaturwissenschaftlerin Vera Nünning (2013, 91-94) charakterisiert die folgenden sieben Merkmale, mit denen Erzählungen Bedeutung zugeschrieben wird und die ebenfalls innerhalb der Moralischen Wochenschriften relevant sein werden: (1) Die Kommunikationssituation ist wichtig zu beachten: Wann, wo, wem und in welchem Register wird etwas erzählt? (2) Es wird eine Welt entworfen, in der sich die Rezipierenden zurechtfinden, die Figuren aufweist und in der Ereignisse stattfinden. (3) Im Allgemeinen weisen Erzählungen Linearität, Sequenzialität und Kontinuität in der Ereignisdarstellung und -verknüpfung auf. (4) Erzählungen sind meist durch einen subjektiven Erfahrungsgehalt der Erzählenden gekennzeichnet. (5) Die Vermittlungsinstanz beziehungsweise die Erzählperspektive lässt Rückschlüsse auf die Vorstellungswelt, die Werte und Denkweisen der Erzählenden zu. (6) Erzählungen können implizit Werte und moralische Positionen vermitteln, die sich zum Beispiel allein schon durch die Disposition der Figuren offenbaren. (7) Erzählungen werden je nach Textsorte und Medium unterschiedlich strukturiert. Es gibt keinen Prototyp der Erzählung, sondern alle Charakteristika werden entsprechend der Textsorte auf verschiedene Weise realisiert. Je nach Textsorte sind Erzählungen inhaltlich, strukturell und sprachlich unterschiedlich aufgebaut, haben andere Ausdrucks- und Sinnstiftungsmöglichkeiten, weshalb Gattungskonventionen bei der Auseinandersetzung mit Erzählungen immer bedacht werden müssen.

In kulturwissenschaftlichem Sinn sind Erzählungen - als Produkte des Erzählens „nicht bloß eine literarische Form oder ein Ausdrucksmedium, sondern ein phänomenologischer und kognitiver Modus der Selbst- und Welterkenntnis“13 (Nünning/

Die Darstellung einer Entwicklung vom Anfang bis zum Ende ist dabei nicht vorhersehbar, sondern verläuft oftmals über unerwartete Brüche und Richtungsänderungen. Die Handlung drängt jeweils zu einem Höhe- und Wendepunkt (Peripetie), an dem eine Lösung und Klärung des Konflikts gegeben wird [kursiv im Orig.]“ (Fahrenwald 2011, 89).

13 „Die Erkenntnis, dass Erzählungen nicht bloß eine literarische Form oder ein Ausdrucksmedium, sondern ein epistemologischer und kognitiver Modus der Selbst- und Welterkenntnis sind, wird sowohl von Jerome Bruner und anderen narrativen Psychologen als auch von Repräsentanten der ,narrativistischen“ Schule von Historikern und Geschichtstheoretikern geteilt. Hayden White bringt die Einsicht in die Semantisierung narrativer Formen prägnant 
Nünning 2002, 2). Der Akt des Erzählens und die Erzählungen selbst dienen zur Organisation von persönlichen Erfahrungen und Ereignissen. Erst im Prozess des Nacherzählens unseres Lebens ordnen wir Erlebtes, geben wir ihm eine Form, eine Struktur und eine Bedeutung (cf. Nünning/Nünning 2010, 12). Erzählungen befähigen uns, die Erfahrungen unseres Lebens in einen zeitlichen Bezug zu setzen und uns somit in der Zeit zu verorten. Dadurch ermöglichen sie uns, unsere individuelle Identität zu konstruieren und uns als Teil eines kollektiven Ganzen wahrzunehmen, in dem jedem Individuum bestimmte soziale Rollen zugeschrieben werden (cf. Scheffel 2011, 77). Aus evolutionsgeschichtlicher Sicht erlauben Erzählungen dem Menschen die „kognitive[...] Verarbeitung von raum-zeitlichen Daten, sie stellen ein konstruktives Verfahren der Sinnherstellung dar und bilden damit eine wichtige Voraussetzung für seine Orientierung in der Welt"“ (ibid., 77).

Als universelle Praxis spielt Erzählen nicht nur bei der Bildung von individuellen, sondern auch von kollektiven Identitäten, bei der Ordnung von Erfahrungen, beim Erinnern, beim Aushandeln von Werten oder bei der Erzeugung von Weltversionen eine wichtige Rolle. Werner Wolf (2013) betont, dass mit dem Erzählen ,eine sinnvolle Repräsentation und eine sozial orientierte Kommunikation von in und durch die Zeit Erlebtem“ (Wolf 2013, 61) angestrebt wird. ${ }^{14}$ Insbesondere weist er darauf hin, dass

auf den Begriff, wenn er bemerkt, ,daß die Erzählung nicht nur eine neutrale diskursive Form ist, die bei der Darstellung realer Ereignisse im Sinne von Entwicklungsprozessen angewandt oder auch nicht angewandt werden kann, sondern vielmehr ontologische und epistemologische Wahlmöglichkeiten mit eindeutig ideologischen und sogar spezifisch politischen Implikationen nach sich zieht" (Nünning A. 2013, 33).

14 Ähnliches postuliert Ottmar Ette, wenn er Literatur wiederholt als ,Lebenswissen “ bezeichnet (cf. Ette 2010; id. 2013, 47-49; Asholt/Ette 2010). Für ihn liegt die Leistung der Literatur im direkten Zugang, den sie zu einer Gesellschaft und ihrer Kultur bereitstellt, denn mit ihrer Hilfe kann das Andere (die andere Gesellschaft und Kultur) über dessen Selbstwahrnehmung entdeckt und dabei gleichzeitig der Blick des Anderen auf das Eigene erkannt werden. Ette unterstreicht dabei die herausragende Fähigkeit der Literatur, ,ihr Wissen als ein Erlebniswissen bereitzustellen, das Schritt für Schritt nachvollzogen [...] werden kann, [...] [und] über große räumliche und zeitliche Distanzen hinweg Menschen erreichen und wirksam [sein kann]“ (Ette 2010, 43). Daneben stellt sie vielfältige Interpretationsmöglichkeiten bereit und wird zu einer „Spielfläche des Viellogischen“ (ibid.; cf. Ette 2013, 53-57), auf der die unterschiedlichsten Wissensinhalte getestet, immer wieder neu miteinander kombiniert und zu neuen Erkenntnissen verarbeitet werden. Literatur bietet somit den Rahmen, in dem verschiedene kulturelle, gesellschaftliche, politische oder psychologische Kontexte und Logiken erprobt und weiterentwickelt werden. Durch diesen viellogischen Aufbau ist Literatur für Ette ,ein Wissen in Bewegung“ (2010, 43), das in ihr ,,aufbewahrt, aber nicht aufgebahrt“ (2013, 53), sondern ,unablässig transformiert“ (ibid.) wird.

Jens Brockmeier und Rom Harré (2005) verweisen ebenfalls darauf, dass in der westlichen Tradition Literatur seit der Renaissance als „Versuchslabor menschlicher Erfahrungen [...] betrachtet worden [ist]. [...] Seit Shakespeare ist Literatur der Ort, an dem menschliche Wirklichkeiten und Möglichkeiten, Faktisches und Fiktives, Realistisches und Imaginäres entworfen und ausprobiert werden, [...]. Die Vorstellung von Literatur als Versuchslabor des 
es in einer Welt ohne Erzählungen weder Kunst noch Medien gäbe, also keine Romane, keine Zeitschriften, kein Theater, keine Computerspiele, keine Comics, keine Talkshows etc.; historische Diskurse ${ }^{15}$ wären inexistent. Alle uns bekannten Schulfächer, in denen Kunst-, Literatur-, Musik-, Rechts-, Medizin-, Kultur-, Sozialgeschichte etc. gelehrt wird, gäbe es nicht. Was bliebe, wären Diskurse, die ohne Erzählen auskommen, wie Beschreibungen, Argumentationen, Handlungsanleitungen etc., die deskriptiven Naturwissenschaften, die Mathematik oder die Logik. Ohne Geschichte und Geschichten gäbe es somit wahrscheinlich keine Kriege, da den Menschen die Erinnerung an den Konflikt fehlte, aber die modernen zivilisatorischen Errungenschaften würden bestehen bleiben. Die Beseitigung von Geschichte und Geschichten würde aber auch bewirken, dass Menschen identitätslos umherirren würden, kaum kommunizieren würden, alle Angst voreinander hätten und die Kriminalität anstiege, weil die lebensweltliche Funktion des Erzählens verloren gegangen wäre (cf. Wolf 2013, 57-60).

Obwohl es zu jeder Epoche und in jeder Kultur Erzählungen gab und gibt, variieren diese - wie die vorherrschenden Weltbilder - von einer Epoche und einer Kultur zur anderen (cf. Nünning/Nünning 2010, 6). Ansgar Nünning schreibt dem Erzählen folglich eine performative Kraft zu, die er darin begründet sieht,

dass Erzählungen nicht bloß ein Medium der Repräsentation kultureller Phänomene sind, sondern auch der Konstruktion von Geschichten, Identitäten und Welten. Erzählungen können zum einen eine wirklichkeitsstrukturierende Funktion erfüllen [...], aber auch Welten und Wirklichkeitsmodelle erzeugen, die so kohärent, ausdrucksstark und suggestiv sein können, dass sie die reale Wirklichkeitserfahrung von Menschen beeinflussen und sogar prägen [...] (Nünning A. 2013, 4; cf. Nünning/Nünning 2010, 12). ${ }^{16}$

Über die Literatur-, Kultur- und Sozialwissenschaften hinaus haben mittlerweile auch die Neurowissenschaften die Leistung von Erzählen und Erzählungen anerkannt. Die australischen Neurowissenschaftler Bem Le Hunte und Jan A. Golembiewski (2014) erläutern in ihrem Artikel „Stories Have the Power to Save us“ das Verdienst von Erzählungen in ähnlicher Weise, wie die genannten Narratolog*innen: Sie erklären, dass das menschliche Gehirn die Fähigkeit entwickelt hat, aus Erzählungen von Anderen und über Andere Bedeutungen und Handlungsanleitungen abzuleiten. Der große

Menschlichen beinhaltet eine weitere Annahme: Nicht nur die Bühne, der Text selbst wird zum Modell der Welt“" (Brockmeier/Harré 2005, 50).

15 Der US-amerikanische Historiker und Literaturwissenschaftler Hayden White postuliert bereits Anfang der 1970er-Jahre, dass Geschichtsschreibung von Erzählungen geprägt ist, und erarbeitet in Metahistory. Die historische Einbildungskraft im 19. Jahrhundert in Europa ein ganzes System narrativer und kognitiver Formen, das allerdings von White nicht systematisch weiterentwickelt oder auf andere Texte angewandt worden ist.

16 Im selben Artikel führt Ansgar Nünning die lebensweltlichen Funktionen des Erzählens weiter aus, wozu er die Episodenbildung, Kohärenzstiftung, Geschehensintegration und Sinnbildung zählt. Auch er veranschaulicht, dass Erzählen Erfahrungen strukturieren und Wissen ordnen kann, und betont seine Möglichkeit, eine Vermittlungsfunktion in der kulturellen Kommunikation, beim Erfahrungsaustausch und der Erzeugung von Intersubjektivität einzunehmen (cf. Nünning A. 2013, 40-45). 
evolutionäre Vorteil, der sich für den Menschen daraus ergibt, ist, dass er dadurch nicht alles selbst durchleben muss, sondern sich über Erzählungen in andere Menschen und Situationen hineinversetzen kann. Dabei erscheint es nicht ausschlaggebend, ob die erzählten Geschichten wahr oder erfunden sind, ${ }^{17}$ entscheidend ist, dass sie für das menschliche Gehirn eine ähnlich ,reale‘ Lebenswelt schaffen, über die sich der/die Empfänger*in in die Lage der dargestellten Person versetzen und somit Empathie für diese entwickeln kann. Die Fähigkeit des Erzählens erscheint den Neurowissenschaftlern also ganz wesentlich für die Selbstdarstellung und -konstruktion von Menschen und vom Menschsein: ,Storytelling, [...], is essential to the way we construct our humanity“ (Le Hunte/Golembiewski 2014, 75). ${ }^{18}$ Diese Lernfähigkeit ist zudem nicht nur auf mündliches Erzählen beschränkt, sondern gilt auch für schriftliches und bildliches Erzählen in und über verschiedene mediale Kanäle hinweg.

Die Literaturwissenschaftler Christian Klein und Matías Martínez (2009) subsumieren die wirklichkeitserzeugenden und -strukturierenden Funktionen des Erzählens unter dem Begriff der ,Wirklichkeitserzählungen', deren wesentliches Merkmal auch sie im konkreten Bezug auf die menschliche Lebenswelt sehen. Durch diesen Bezug auf reale, räumlich und zeitlich konkrete Sachverhalte sowie Ereignisse erheben Wirklichkeitserzählungen einen ,faktualen Geltungsanspruch', durch den sich drei modellhafte Typen von Wirklichkeitserzählungen unterscheiden lassen: ${ }^{19}$ (1) deskriptive, (2) normative und (3) voraussagende Wirklichkeitserzählungen. (1) Die Funktion der des-

17 Ebenso sehen Christian Klein und Matías Martínez (2009) faktuale wie fiktionale Erzählungen als Teile einer real stattfindenden Kommunikation. Für sie liegt der einzige Unterschied darin, dass letztere komplexer sind, ,weil sie außer der realen auch noch eine zweite, imaginäre Kommunikationssituation gestalten. In dieser zweiten Situation kommt ein erfundener Erzähler zu Wort (z. B. der fiktive Ich-Erzähler [...]). Die fiktionale Erzählung enthält also sowohl eine reale wie eine imaginäre Kommunikation und stellt insofern eine ,kommunizierte Kommunikation“ dar“ (Klein/Martínez 2009, 2).

18 Wenn Peter Salovey (2017), Sozialpsychologe und Präsident der Yale University, in seinem auf den Seiten des Weltwirtschaftsforums (das alljährlich im schweizerischen Davos stattfindet) veröffentlichten Artikel die Wichtigkeit der Geisteswissenschaften hervorhebt, so tut er dies auch mit Bezug auf das Erzählen. Durch das Lesen von Büchern - ebenso wie durch das Hören von Musik, das Sehen von Theaterstücken oder das Betrachten von Bildern in einem Museum - wird, so betont Salovey, unsere emotionale Intelligenz geschult und wir erlernen wichtige Fähigkeiten wie Empathie und Vorstellungskraft.

In dieselbe Kerbe schlägt die ehemalige kanadische Botschafterin in Österreich, Ingrid Hall, in ihrer Eröffnungsrede anlässlich des fünfjährigen Bestehens des Zentrums für Kanada-Studien in Innsbruck, wenn sie konzediert, dass die Geisteswissenschaften auch im 21. Jahrhundert ausschlaggebend sein werden, um Themen wie sexuelle, religiöse und ethnische Diskriminierung, Xenophobie, Intoleranz und Hass, aber auch Möglichkeiten der Armutsbekämpfung, des Ausbaus der medizinischen Grundversorgung und der Erhöhung der Alphabetisierungsrate besser zu verstehen und Strategien dagegen bzw. dafür zu entwickeln (cf. Hall 2003, 51).

19 In ähnlicher Weise konstatieren Jens Brockmeier und Rom Harré die Funktionen von Erzählungen als deskriptiv, analytisch und normativ, siehe Brockmeier/Harré $(2005,46)$ „Die narrative Wende: Reichweite und Grenzen eines alternativen Paradigmas“. 
kriptiven Wirklichkeitserzählungen liegt in der Schilderung von Ereignissen, die sich tatsächlich zugetragen haben, woraus sich ein Geltungsanspruch gemäß den beiden Polen ,wahr' versus ,falsch ‘ ableitet, wie dies in der Historiografie oder im Journalismus im Zuge der Rekonstruktion von Ereignissen der Fall ist. (2) Die Funktion normativer Wirklichkeitserzählungen bezieht sich auf die Beschreibung einer erwünschten Wirklichkeit. Damit wird das Ziel verfolgt, ,eine bestimmte (gesellschaftliche oder individuelle) Praxis zu regulieren, das geschieht durch exemplifikatorische Darstellungen (menschlicher Handlungen). Der Geltungsanspruch orientiert sich an der Dichotomie ,richtig handeln vs. falsch handeln““(Klein/Martínez 2009, 6). Als Beispiele für normative Wirklichkeitserzählungen führen Klein und Martínez Verhaltensratgeber, moralische Handlungsnormen sowie juristische Gesetze an. (3) Die Funktion voraussagender Wirklichkeitserzählungen liegt schließlich in der Schilderung einer erwarteten künftigen Wirklichkeit, für die allgemeine Strukturmerkmale bereits festgelegt worden sind, wie bei medizinischen Prognosen oder naturwissenschaftlich fundierten Vorhersagen zum Klimawandel. Der hier erhobene Geltungsanspruch richtet sich dabei an den beiden Polen ,plausibel` versus ,nicht plausibel' aus.

Wie zu zeigen sein wird, stellen auch die erzählten Inhalte der Moralischen Wochenschriften durch ihren ausgeprägten Bezug zur menschlichen Lebenswelt einen deskriptiven Geltungsanspruch, der aufgrund der sich erst ausbildenden bürgerlichen Gesellschaftsschicht für die Leser*innen zusätzlich normative und voraussagende Geltungsansprüche einnimmt.

\subsection{Mediale Wissens- Und Welterzeugung}

Kulturelles Wissen wird über die (Massen-)Medien generiert und disseminiert. Die in ihnen verhandelten Diskurse lenken unsere Wahrnehmung und unser Bewusstsein und nehmen somit Einfluss auf das gesellschaftliche Zusammenleben. Das Leistungsvermögen von literarischen Texten als Medien des kollektiven Gedächtnisses von Kulturen hat Astrid Erll (2005) in die Bereiche der Speicherung, der Zirkulation und des Abrufs von Wissen gegliedert. In Anlehnung an Maurice Halbwachs, der Erinnerungen stets in Abhängigkeit der ,cadres sociaux ' (sozialen Rahmungen) begreift (cf. Halbwachs 1925), spricht Erll von den ,cadres médiaux‘ (medialen Rahmungen), die auf die Erinnerung einer Person oder einer Gruppe einwirken. Erst durch mediale Repräsentationen können wir auf das spezifische Wissen von Gruppen und deren Vorstellungswelt zugreifen. Die ,medialen Rahmen des Erinnerns' ermöglichen uns, eigene und fremde Erfahrungen ins Bewusstsein zu rufen und zu deuten. Sie beeinflussen und präformieren unsere Wahrnehmung und steuern unseren Erinnerungsabruf. Ähnlich wie im vorherigen Abschnitt über das Erzählen hervorgehoben wurde, kann Literatur - die schließlich aus Erzählungen besteht - als Speichermedium einer Erinnerungskultur normativ und formativ rezipiert werden und somit eine kulturelle Dimension annehmen. Als Zirkulationsmedium konstruiert und vermittelt Literatur Inhalte des kollektiven Gedächtnisses (wie Geschichtsbilder, Werte, Normen, Identitäten), wobei wir diesen Inhalten eine gewisse Realitätsnähe zugestehen müssen, um sie als glaubhaft und normativ aufzufassen. Als Abrufmedium funktioniert Literatur schließlich dann, wenn wir allein durch die Nennung von bestimmten Texten an bestimmte Geschichtsbilder, Werte oder Normen denken (cf. Erll 2005, 254-263). 